\title{
CONTACT HOMOLOGY OF BRIESKORN MANIFOLDS
}

\author{
OTTO VAN KOERT
}

\begin{abstract}
We give an algorithm for computing the contact homology of some Brieskorn manifolds. Brieskorn manifolds can be regarded as circle-bundles over orbifolds and our algorithm expresses cylindrical contact homology of the Brieskorn manifold in terms of the homology of the underlying orbifold. As an application, we construct infinitely many contact structures on the class of simply connected contact manifolds that admit nice contact forms (i.e. no Reeb orbits of degree $-1,0$ or 1 ) and have index positivity with trivial first Chern class.
\end{abstract}

\section{INTRODUCTION}

For a long time it has been known that contact manifolds may carry many nonisomorphic contact structures. A first way to distinguish these structures from each other is by considering their Chern class or their formal homotopy class. In dimension 3 we can, in addition, sometimes distinguish contact structures by showing tightness or overtwistedness as was shown first by Bennequin. At present, the latter two notions do not have generalizations to higher dimensions.

Eliashberg was the first to show that the classical invariants (i.e. the Chern class and formal homotopy class) are not always enough for a full classification of contact structures on a given manifold in dimension larger than 3. He showed that spheres in dimensions $4 n+1$ admit a non-standard, yet homotopically trivial contact structure in [4. Giroux gave an interesting example in dimension 3 in [7. He exhibited infinitely many tight contact structures on $T^{3}$ in the same homotopy class of plane fields. Since then more general techniques to distinguish contact structures were introduced. Eliashberg and Hofer's contact homology is such a technique (for a survey, see [5], and for a more recent description, 6]). It works, roughly speaking, as follows. The Reeb orbits on a given contact manifold can be given a grading. The chain complexes are freely generated by the closed Reeb orbits. Then a differential can be defined by counting certain holomorphic curves asymptotic to Reeb orbits in the symplectization of the contact manifold. It can be shown that the homology of this differential is independent of the contact form under suitable conditions and it is hence an invariant of the contact structure. Since its introduction, several examples of non-isomorphic contact structures in the same homotopy class have been found.

One of these examples is a family of Brieskorn spheres in dimensions $4 n+1$. These were found by Ustilovsky in [14]. In his thesis he used a few other tools that can easily be applied to construct more contact structures on the same manifold. This is done by connect summing. It is well known that the connected sum of two contact manifolds carries again a contact structure, see [11] and [16]. In his thesis

1991 Mathematics Subject Classification. Primary 53D10 .

Key words and phrases. Contact geometry, contact homology. 
Ustilovsky showed that we can roughly see what happens to the resulting contact homology, provided we are given sufficient information on the given two contact manifolds.

In this paper, we use these ideas to construct more contact structures on a certain class of contact manifolds. The starting point is the computation of the contact homology of Brieskorn manifolds. This is done by calculating the MorseBott contact homology, an extension of contact homology due to Bourgeois 2]. It allows a larger class of contact forms to be admissible than the generic contact forms required in the original formulation of contact homology ([6]). Using Bourgeois's techniques we give an algorithm that computes the contact homology of a Brieskorn manifold.

Notice that a Brieskorn manifold can be regarded as an $S^{1}$-bundle over an orbifold. It turns out that the resulting contact homology of the Brieskorn manifold with its natural contact structure can be expressed in terms of the homology of that orbifold and its singular subspaces with some degree shifts that we explicitly compute. By combining this algorithm with Ustilovsky's ideas we produce a large class of contact manifolds with infinitely many non-isomorphic contact structures.

Acknowledgements. This paper is a part of my thesis, which I wrote under supervision of H. Geiges at the university of Cologne. I am indebted to him for his support and patience. I am very grateful to F. Bourgeois for his explanations and for many valuable comments and suggestions. I would also like to thank R. Lipshitz for his comments. Currently I am supported by the F.N.R.S., Belgium.

\section{Preliminaries}

The Brieskorn manifolds $\Sigma\left(a_{0}, \ldots, a_{n}\right) \subset \mathbb{C}^{n+1}$ (with $a_{0}, \ldots, a_{n} \in \mathbb{N}$ ) are defined as the intersection of the sphere $S^{2 n+1}=\left\{\left.\left(z_{0}, \ldots, z_{n}\right) \in \mathbb{C}^{n+1}|| z_{0}\right|^{2}+\right.$ $\left.\ldots+\left|z_{n}\right|^{2}=1\right\}$ with the zero set of the polynomial $f\left(z_{0}, \ldots, z_{n}\right)=z_{0}^{a_{0}}+\ldots+z_{n}^{a_{n}}$. The Brieskorn manifolds carry a contact structure which was shown by Lutz and Meckert [10]. We will take the following contact form on $\Sigma\left(a_{0}, \ldots, a_{n}\right)$ :

$$
\alpha=\frac{i}{8} \sum_{j=0}^{n} a_{j}\left(z_{j} \mathrm{~d} \bar{z}_{j}-\bar{z}_{j} \mathrm{~d} z_{j}\right) .
$$

We will denote the contact structure by $\xi=\operatorname{ker} \alpha$. The Reeb vector field of this contact form has a particularly simple shape

$$
R=4 i\left(z_{0} / a_{0}, \ldots, z_{n} / a_{n}\right),
$$

where we regard $T \Sigma\left(a_{0}, \ldots, a_{n}\right)$ as a subset of $T \mathbb{C}^{n+1}$. The Reeb flow then looks like

$$
\varphi_{t}(z)=\left(e^{4 i t / a_{0}} z_{0}, \ldots, e^{4 i t / a_{n}} z_{n}\right)
$$

which means that all Reeb orbits are closed. In particular, all Reeb orbits are degenerate. We can, however, still compute the contact homology using the MorseBott approach due to Bourgeois [2, without an explicit perturbation of the contact form. In the rest of this section we will establish some notation and set up some ingredients of the Morse-Bott approach.

The first step will be the computation of the Maslov indices of the Reeb orbits. They will give the grading of the Morse-Bott complex. To compute the Maslov indices we will be using the same method as Ustilovsky did in 14: we extend the 
Reeb field so that it gives rise to a symplectomorphism of $\mathbb{C}^{n+1}$. We may then do the computations in $\mathbb{C}^{n+1}$. In our discussion, the symplectic form on $\mathbb{C}^{n+1}$ is given by

$$
\omega=\mathrm{d} \alpha=\frac{i}{4} \sum_{j=0}^{n} a_{j} \mathrm{~d} z_{j} \wedge \mathrm{d} \bar{z}_{j} .
$$

We start by showing that we can construct a basis of the symplectic complement $\xi^{\omega}$ of $\xi$ in $\mathbb{C}^{n+1}$. Notice that $\xi^{\omega}=\operatorname{span}\left(X_{1}, Y_{1}, X_{2}, Y_{2}\right)$, where

$$
\begin{aligned}
X_{1}=\left(\bar{z}_{0}^{a_{0}-1}, \ldots, \bar{z}_{n}^{a_{n}-1}\right), & Y_{1}=i X_{1}, \\
X_{2}=-2 i\left(\frac{z_{0}}{a_{0}}, \ldots, \frac{z_{n}}{a_{n}}\right), & Y_{2}=\left(z_{0}, \ldots, z_{n}\right) .
\end{aligned}
$$

We are going to use a "Gram-Schmidt" process to turn this into a symplectic standard basis. This new basis is given by

$$
\begin{aligned}
\tilde{X}_{1}=\frac{X_{1}}{\sqrt{\omega\left(X_{1}, Y_{1}\right)}}, & \tilde{Y}_{1}=\frac{Y_{1}}{\sqrt{\omega\left(X_{1}, Y_{1}\right)}} \\
\tilde{X}_{2}=X_{2}, & \tilde{Y}_{2}=Y_{2}-\frac{\omega\left(X_{1}, Y_{2}\right) Y_{1}-\omega\left(Y_{1}, Y_{2}\right) X_{1}}{\omega\left(X_{1}, Y_{1}\right)}=Y_{2}-\frac{\sum a_{i} z_{i}^{a_{i}}}{2 \omega\left(X_{1}, Y_{1}\right)} X_{1},
\end{aligned}
$$

where we have used that $\omega\left(X_{1}, Y_{1}\right)=\frac{1}{2} \sum_{j} a_{j}\left|z_{j}\right|^{2\left(a_{j}-1\right)}>0$. This new basis is a standard symplectic basis of $\xi^{\omega}$. Note that $\xi \oplus \xi^{\omega}=\mathbb{C}^{n+1}$.

This computation gives us a bonus: $c_{1}(\xi)=0$. Indeed, both $\xi^{\omega}$ and $\mathbb{C}^{n+1}$ are symplectically trivial and have therefore trivial total Chern class. Hence the Chern class of $\xi$ is trivial as well.

2.1. Maslov indices. The Reeb flow $\varphi$ which we introduced in the previous section can obviously be extended to a flow on $\mathbb{C}^{n+1}$, which we will also denote by $\varphi$. We find the following for the action of the extended Reeb flow on $\xi^{\omega}$ :

$$
\begin{aligned}
T \varphi_{t}\left(\tilde{X}_{1}(x)\right)=e^{4 i t} \tilde{X}_{1}\left(\varphi_{t}(x)\right), & T \varphi_{t}\left(\tilde{Y}_{1}(x)\right) & =e^{4 i t} \tilde{Y}_{1}\left(\varphi_{t}(x)\right) \\
T \varphi_{t}\left(\tilde{X}_{2}(x)\right)=\tilde{X}_{2}\left(\varphi_{t}(x)\right), & T \varphi_{t}\left(\tilde{Y}_{2}(x)\right) & =\tilde{Y}_{2}\left(\varphi_{t}(x)\right) .
\end{aligned}
$$

The action of $T \varphi_{t}$ on $\mathbb{C}^{n+1}$ is given by the differential of $\varphi_{t}$. In terms of the standard basis of $\mathbb{C}^{n+1}$, it is given by the diagonal matrix

$$
\operatorname{diag}\left(e^{4 i t / a_{0}}, \ldots, e^{4 i t / a_{n}}\right) .
$$

We can now use the additivity of the Maslov index under direct sums of symplectic paths to get the index for $\xi$ (for general properties of the Maslov index see [13] and [15]). Let $\gamma=\left\{\varphi_{t}(p) \mid t \in[0, T]\right\}$ be a closed Reeb orbit of period T through $p \in \Sigma\left(a_{0}, \ldots, a_{n}\right)$. Let $\Phi_{\mathbb{C}^{n+1}}(t)=T \varphi_{t}(p)$ for $t \in[0, T]$, the path of symplectic matrices associated to the Reeb flow extended to $\mathbb{C}^{n+1}$. Write $\Phi_{\xi^{\omega}}(t)=\left.T \varphi_{t}(p)\right|_{\xi^{\omega}}$ for $t \in[0, T]$, the path of symplectic matrices induced by the Reeb flow on the symplectic complement of $\xi$. Then

$$
\mu(\gamma)=\mu\left(\Phi_{\mathbb{C}^{n+1}}\right)-\mu\left(\Phi_{\xi^{\omega}}\right)
$$

Note also that the right-hand side of the above equation can be easily computed using additivity of the Maslov index under direct sums and the following formula (see [15]):

$$
\mu\left(\left.e^{i t}\right|_{t \in[0, T]}\right)=\left\{\begin{array}{cl}
\frac{T}{\pi} & \text { if } T \in 2 \pi \mathbb{Z} \\
2\left\lfloor\frac{T}{2 \pi}\right\rfloor+1 & \text { otherwise. }
\end{array}\right.
$$


2.2. Homology of Brieskorn manifolds. In [12 Randell proves an algorithm that computes the homology of generalized Brieskorn manifolds. Since we are here only interested in the homology of Brieskorn manifolds, we list the algorithm in the Brieskorn case and refer the reader to 12 for further details. Let $M=\Sigma\left(a_{0}, \ldots, a_{n}\right)$. For convenience, we introduce the following notation. Let $I$ denote the set $\{0, \ldots, n\}$. A subset of $I$ with $s$ elements will by denoted by $I_{s}$. If $I_{s}=\left\{i_{1}, \ldots, i_{s}\right\}$, then let $K\left(I_{s}\right)$ denote the Brieskorn manifold $\Sigma\left(a_{i_{1}}, \ldots, a_{i_{s}}\right)$ of dimension $2 s-3$. Note that $M=K(I)$ contains all manifolds $K\left(I_{s}\right)$ for all $1<s \leq n+1$ in a natural way by restricting to suitable coordinate hyperplanes. We define following Randell

$$
\kappa\left(K\left(I_{s}\right)\right)=\sum_{I_{t} \subset I_{s}}(-1)^{s-t} \frac{\prod_{i \in I_{t}} a_{i}}{\operatorname{lcm}_{j \in I_{t}} a_{j}} .
$$

Then we have for the free part of the homology

$$
\operatorname{rk} \tilde{H}_{n-1}(M, \mathbb{Z})=\kappa(K(I)) .
$$

For the torsion part, a few additional definitions are required. We set

$$
k\left(K\left(I_{s}\right)\right)= \begin{cases}\kappa\left(K\left(I_{s}\right)\right) & \text { if } n+1-s \text { is odd } \\ 0 & \text { otherwise. }\end{cases}
$$

Let $C(\emptyset)=\operatorname{gcd}_{i \in I} a_{i}$ and set

$$
C\left(I_{s}\right)=\frac{\underset{i \in\left(I-I_{s}\right)}{\operatorname{gcd}} a_{i}}{\prod_{I_{t} \varsubsetneqq I_{s}} C\left(I_{t}\right)} .
$$

Now set $d_{j}=\prod_{k\left(K\left(I_{s}\right)\right) \geq j} C\left(I_{s}\right)$ and $r=\max \left\{k\left(K\left(I_{s}\right)\right) \mid I_{s} \subset I\right\}$. Then we have

$$
\operatorname{Tor} H_{n-1}(K(I), \mathbb{Z})=Z_{d_{1}} \oplus \ldots \oplus Z_{d_{r}} .
$$

There is another interesting result from Randell's paper that we will use. Set $d=\operatorname{lcm}\left(a_{0}, \ldots, a_{n}\right)$ and $q_{i}=d / a_{i}$. The Brieskorn manifolds admit the following $S^{1}$-action:

$$
t\left(z_{0}, \ldots, z_{n}\right)=\left(t^{q_{0}} z_{0}, \ldots, t^{q_{n}} z_{n}\right)
$$

for $t \in S^{1}$. Then define $M^{*}:=M / S^{1}$, which, in general, will be an orbifold. It is important to note here that this $S^{1}$-action coincides with the flow of the Reeb field. Again from [12] we can compute the rational homology of $M^{*}$. The result is

$$
H_{q}\left(M^{*}, \mathbb{Q}\right) \cong\left\{\begin{array}{l}
\mathbb{Q}, q \text { even } 0 \leq q \leq \operatorname{dim} M^{*} \\
0, \text { otherwise }
\end{array}\right\} \oplus\left\{\begin{array}{l}
\mathbb{Q}^{\kappa}, q=\frac{1}{2} \operatorname{dim} M^{*} \\
0, \text { otherwise }
\end{array}\right\}
$$

where $\kappa=\kappa(K(I))$.

2.3. Morse-Bott contact homology. Let $M$ denote a manifold with contact structure $\xi$ which is given by as the kernel of the 1 -form $\alpha$. Define the action functional

$$
\begin{aligned}
\mathcal{A}: C^{\infty}\left(S^{1}, M\right) & \rightarrow \mathbb{R} \\
\gamma & \mapsto \int_{\gamma} \alpha .
\end{aligned}
$$

The critical points of this action functional are the periodic orbits of the Reeb flow $\varphi_{t}$. Cylindrical contact homology can then be regarded as a kind of Morse theory for this action functional, where one needs appropriate regularity conditions. For 
instance one would need a generic contact form for which all Reeb orbits are nondegenerate. In particular this means that symmetric contact forms like the one for the Brieskorn manifolds are excluded. This makes computations in general quite hard. The Morse-Bott approach to contact homology due to Bourgeois is often much better suited to symmetric contact forms. We will now summarize some of Bourgeois's results from [2. As we need some of his lemmas later on, we will list those along with one of the main theorems of 2. We will restrict in some of his theorems to contact forms for which all Reeb orbits are closed. This is not a requirement in Bourgeois's work and only takes care of a small technical detail.

Let $\sigma(\alpha)$ denote the action spectrum of $\alpha$, i.e. the critical values of the action functional $\mathcal{A}$.

Definition 2.1. A contact form $\alpha$ is said to be of Morse-Bott type if the action spectrum $\sigma(\alpha)$ is discrete and if, for every $T^{\prime} \in \sigma(\alpha), M_{T^{\prime}}=\left\{p \in M \mid \varphi_{T^{\prime}}(p)=p\right\}$ is a closed, smooth submanifold of $M$, such that the rank $\left.d \alpha\right|_{M_{T^{\prime}}}$ is locally constant and $T_{p} M_{T^{\prime}}=\operatorname{ker}\left(T \varphi_{T^{\prime}}-i d\right)_{p}$.

The Reeb flow induces an $S^{1}$-action on $M_{T^{\prime}}$. Using this action we define the orbit space $S_{T^{\prime}}:=M_{T^{\prime}} / S^{1}$. Note that these orbit spaces are orbifolds in general.

The chains of the Morse-Bott chain complex will correspond to the critical points of suitable Morse functions on the orbit spaces. Bourgeois constructs these Morse functions by induction (note that he needs Morse functions on orbifolds for which he introduces a suitable notion in [2]). We will now describe his construction.

For the smallest $T \in \sigma(\alpha)$, the orbit space $S_{T}$ is a smooth manifold. Take any Morse function $f_{T}$ on it. For larger $T \in \sigma(\alpha), S_{T}$ is an orbifold where the singularities are the orbit spaces $S_{T^{\prime}}$ with $T^{\prime}$ dividing $T$. The previously defined Morse functions $f_{T^{\prime}}$ on the orbit spaces $S_{T^{\prime}}$ are extended to a function $f_{T}$ on $S_{T}$ by requiring that the Hessian of $f_{T}$ restricted to the normal bundle of $S_{T^{\prime}}$ is positive definite. These Morse functions are then lifted to $M_{T}$ and extended to a function $\bar{f}_{T}$ on $M$ such that they have support only in a tubular neighborhood of $M_{T}$.

For $T \in \sigma(\alpha)$ Bourgeois considers the following family of contact forms $\alpha_{\lambda}=$ $\left(1+\lambda \bar{f}_{T}\right) \alpha$. We have

Lemma 2.2 (Bourgeois). For all $T$, we can choose $\lambda>0$ small enough such that the periodic orbits of $R_{\alpha_{\lambda}}$ in $M$ of action $T^{\prime} \leq T$ are non-degenerate and correspond to the critical points of $f_{T^{\prime}}$.

Let $p \in S_{T^{\prime}}$ be a critical point of $f_{T^{\prime}}$ and denote its corresponding closed Reeb orbit (and its multiple covers) by $\gamma_{k T^{\prime}}^{p}$ for $k=1,2, \ldots$. As Bourgeois's construction is explicit enough, he is able to compute the Conley-Zehnder indices of these Reeb orbits for small $\lambda$ such that Lemma 2.2 applies:

$$
\mu_{C Z}\left(\gamma_{k T^{\prime}}^{p}\right)=\mu\left(S_{k T^{\prime}}\right)-\frac{1}{2} \operatorname{dim} S_{k T^{\prime}}+\operatorname{index}_{p}\left(f_{k T^{\prime}}\right) .
$$

This determines the degree of the Reeb orbits associated with the perturbed contact form with small period. The following notion is helpful in dealing with orbits of larger period.

Definition 2.3. The orbit spaces $S_{T}$ are said to have index positivity if there exist constants $c>0$ and $c^{\prime}$ such that $\mu\left(S_{T}\right)>c T+c^{\prime}$ for all $T \in \sigma(\alpha)$. 
Similarly, we define index negativity of the orbit spaces $S_{T}$ if there are constants $c<0$ and $c^{\prime}$ such that $\mu\left(S_{T}\right)<c T+c^{\prime}$. In order to control the behavior of orbits with larger period, Bourgeois has the following result.

Lemma 2.4 (Bourgeois). Assume that the orbit spaces $S_{T}$ have index positivity, that $c_{1}(\xi)=0$ and that all Reeb orbits are closed. Then there exists a $\lambda_{0}>0$ such that, if $0<\lambda<\lambda_{0}$, all period orbits $\gamma_{\lambda}$ of $R_{\alpha_{\lambda}}$ of action greater than $T$ satisfy $\mu_{C Z}\left(\gamma_{\lambda}\right)>\frac{c}{2} T$, where $c$ is the positive constant from the index positivity of $S_{T}$.

This lemma makes sure that other closed Reeb orbits that do not correspond to any critical point of the Morse functions on the orbit spaces have large Maslov indices. A similar result holds in case of index negativity of the orbit spaces.

The chains of the Morse-Bott complex are the critical points $p$ of the Morse functions $f_{T}$ for $T \in \sigma(\alpha)$ with degree given by

$$
\operatorname{deg}(p)=\mu\left(S_{k T^{\prime}}\right)-\frac{1}{2} \operatorname{dim} S_{k T^{\prime}}+\operatorname{index}_{p}\left(f_{k T^{\prime}}\right)+n-3 .
$$

2.3.1. Moduli spaces of generalized holomorphic curves. Before we come the definition of the moduli spaces, first recall that the fibered product of $A$ and $B$ over $C$ for maps $f: A \rightarrow C, g: B \rightarrow C$ is given by

$$
A \times_{C} B=\{(a, b) \in A \times B \mid f(a)=g(b)\} .
$$

Let $\mathcal{M}\left(S^{+}, S^{-}\right)$denote the moduli space of holomorphic curves with degenerate asymptotics, with orbit spaces $S^{+}$and $S^{-}$for the positive and negative puncture, respectively. In other words, elements of $\mathcal{M}\left(S^{+}, S^{-}\right)$correspond to holomorphic cylinders that are asymptotic to a closed Reeb orbit in $S^{+}$at the positive end and asymptotic to a closed Reeb orbit in $S^{-}$at the negative end of the cylinder. In special cases, such as simple holomorphic curves (which for instance happens if the orbits in $S^{+}$and $S^{-}$are $k$ - and $l$-fold covers of simple Reeb orbits and $k$ and $l$ are relatively prime) one can choose the almost complex structure in such a way that the moduli space is a smooth manifold. In general the moduli space of holomorphic curves is not a smooth manifold though.

In the Morse-Bott picture we shall make use of the moduli space of generalized holomorphic cylinders given by

$$
\mathcal{M}^{f_{T}}\left(S^{+}, S^{-}\right)=\mathcal{M}\left(S^{+}, S^{-}\right) \bigcup\left(\mathcal{M}\left(S^{+}, S^{\prime}\right) \times{ }_{S^{\prime}}\left(\mathbb{R}^{+} \times \mathcal{M}\left(S^{\prime}, S^{-}\right)\right)\right) \bigcup \ldots
$$

where the union runs over successive fibered products. Note that the union is finite, because a holomorphic curve has to have positive energy (the energy is equal the action of the top Reeb orbit minus the action of the bottom Reeb orbit) and the action spectrum is discrete. The projection maps for these fibered products are $e v^{-}$ and $\varphi^{f_{T}} \circ e v^{+}$. The maps $e v^{-}$and $e v^{+}$are the evaluation maps at the negative and positive puncture, respectively, i.e. $e v^{ \pm}: \mathcal{M}\left(S^{+}, S^{-}\right) \rightarrow S^{ \pm}$and here $\varphi^{f_{T}}$ is the gradient flow of $f_{T}$ on the orbit space. So $\varphi^{f_{T}} \circ e v^{+}:(t, u) \mapsto \varphi^{f_{T}}(t)\left(e v^{+}(u)\right)$, where $t \in \mathbb{R}^{+}$and $u \in \mathcal{M}\left(S^{\prime}, S^{\prime \prime}\right)$.

In other words, generalized holomorphic cylinders are "cascades" of holomorphic cylinders and gradient trajectories. For example, the second term of the union consists of a holomorphic cylinder starting asymptotically at a closed Reeb orbit in $S^{+}$and ending (not asymptotically) at a closed Reeb orbit in $S^{\prime}$. After that the cascade follows the gradient flow of the Morse function $f_{T}$ on the orbit space $S^{\prime}$ for a finite time. Finally the cascade continues with a holomorphic cylinder ending 
asymptotically at a closed Reeb orbit in $S^{-}$. For a discussion of the general case (i.e. not only cylinders) see [2] page 38 .

2.3.2. Orientation. The moduli space of holomorphic curves needs to be oriented. Bourgeois does this using the coherent orientation scheme, in a similar way as in the original formulation of contact homology. There are a few obstructions to the existence of coherent orientations. First of all, there is the notion of a bad Reeb orbit. Since we are working here with Morse-Bott instead of generic contact forms, the notion is slightly different from the standard one described in [6].

Definition 2.5. A Reeb orbit $\gamma$ is said to be bad if it is the $2 m$-fold cover of a simple Reeb orbit $\gamma^{\prime} \in S_{T}$ and if

$$
\left(\mu\left(S_{2 T}\right) \pm \frac{1}{2} \operatorname{dim} S_{2 T}\right)-\left(\mu\left(S_{T}\right) \pm \frac{1}{2} \operatorname{dim} S_{T}\right)
$$

is odd. If a Reeb orbit is not bad, it is said to be good.

In Morse-Bott contact homology, there can be another obstruction for coherent orientations. For this, we recall a way the moduli spaces of holomorphic curves can be oriented (see chapter 7 of [2]) in case the asymptotics are non-degenerate. We have a map $\pi$ from the moduli space of holomorphic curves $\mathcal{M}$ to the space of Fredholm operators $\mathcal{O}\left(\gamma^{+}, \gamma^{-}\right)$with behavior near the punctures corresponding to the closed Reeb orbits $\gamma^{+}$and $\gamma^{-}$defined by sending a holomorphic map to its linearized Cauchy-Riemann operator. Let $\mathcal{L}$ denote the determinant bundle over $\mathcal{O}\left(\gamma^{+}, \gamma^{-}\right)$. Then $\pi^{*} \mathcal{L}$ is naturally isomorphic to the top exterior power of $T \mathcal{M}$. In case the asymptotics for $\mathcal{O}\left(\gamma^{+}, \gamma^{-}\right)$are fixed, $\mathcal{O}\left(\gamma^{+}, \gamma^{-}\right)$is contractible and hence $\mathcal{L}$ is trivial. In this case there are no obstructions to orient $\mathcal{M}$.

On the other hand, if the asymptotics are allowed to vary as is the case for the Morse-Bott formalism, then we may get a non-contractible space of Fredholm operators. This can happen, because the space of operators fibers over submanifolds of the form $M_{T}$. If that space $M_{T}$ is not simply connected, it may contain a loop of operators such that the determinant bundle over that loop is not trivial. Such a loop is called a disorienting loop. We should remark here that if the projection of this loop to the orbit space $S_{T}$ is contractible, the loop in $M_{T}$ is homotopic to a bad Reeb orbit.

These phenomena can in general be present, because the linearized CauchyRiemann operator is usually only real linear and not complex linear. In favorable cases, the linearized Cauchy-Riemann operator is complex linear as well and an orientation on the determinant line bundle can be obtained directly from the induced complex structure on the kernel and cokernel of the linearized Cauchy-Riemann operator. This removes the need to see whether there are disorienting loops or bad orbits, because they cannot occur in that case.

2.3.3. Differential for cylindrical contact homology in the Morse-Bott case. With the above remarks in mind, the differential can be defined. The differential of the chain complex for cylindrical contact homology is given by

$$
d p=\partial p+\sum_{q} n_{q} q
$$


where $p \in S_{T}, \partial$ is the Morse-Witten differential of the Morse function $f_{T}$ on $S_{T^{\prime}}$, $q \in S_{T^{\prime}}$ and $n_{q}$ is the algebraic number of elements in the fibered product

$$
\left(W^{u}(p) \times_{S_{T}} \mathcal{M}^{f_{T}}\left(S_{T} ; S_{T^{\prime}}\right) \times_{S_{T^{\prime}}} W^{s}(q)\right) / \mathbb{R}
$$

if this product is zero-dimensional and 0 otherwise $\left(q \in S_{T^{\prime}}\right)$. In this product $W^{s}$ and $W^{u}$ denote the stable and unstable manifolds of a critical point of $f_{T}$ on an orbit space, respectively.

The precise definition of $n_{q}$ is more involved. However, we shall argue that in our case of a Brieskorn manifold and almost complex structure there are no zerodimensional moduli spaces of holomorphic curves. As a result, the second term in the differential vanishes for us.

Theorem 2.6 (Bourgeois). Let $\alpha$ be a contact form of Morse-Bott type for a contact structure $\xi$ on $M$ that satisfies $c_{1}(\xi)=0$. Assume that all Reeb orbits are closed. Assume that, for all $T \in \sigma(\alpha), M_{T}$ and $S_{T}$ are orientable, $\pi_{1}\left(S_{T}\right)$ has no disorienting loop, and all Reeb orbits in $S_{T}$ are good. Assume that the almost complex structure $J$ is invariant under the Reeb flow on all submanifolds $M_{T}$. Assume that the cylindrical homology is well defined: the Morse-Bott chain complex has no contractible orbits of index $-1,0$ or +1 . Assume furthermore that all orbit spaces $S_{T}$ of contractible periodic orbits have index positivity or index negativity. Then the homology $H_{*}\left(C_{*}^{\bar{a}}\right)$ of the Morse-Bott chain complex $\left(C_{*}^{\bar{a}}, d\right)$ of $(M, \alpha)$ is isomorphic to the cylindrical homology $H F_{*}^{\bar{a}}(M, \xi)$ of $(M, \xi)$, where both homologies have coefficients in the Novikov ring of $H_{2}(M, \mathbb{Z}) / \mathcal{R}$.

There are other (improved) versions of this theorem, but this one suffices for our needs. In addition we will take the ring $\mathcal{R}$ in the above theorem to be $H_{2}(M, \mathbb{Z})$, or in other words we will use $\mathbb{Q}$ coefficients for the Morse-Bott chain complex.

\section{Algorithm for the Computation of the Cylindrical homology of SOME BRIESKORN MANIFOLDS}

Consider the Brieskorn manifold $M=\Sigma\left(a_{0}, \ldots, a_{n}\right) \subset \mathbb{C}^{n+1}$ with contact form induced by $\alpha=\frac{i}{8} \sum a_{j}\left(z_{j} \mathrm{~d} \bar{z}_{j}-\bar{z}_{j} \mathrm{~d} z_{j}\right)$ and assume $n \geq 3$, which means that $M$ is at least 5 -dimensional. The Reeb flow of the contact form $\alpha$ is given by $\varphi_{t}(z)=$

$\left(e^{4 i t / a_{0}} z_{0}, \ldots, e^{4 i t / a_{n}} z_{n}\right)$. As we remarked in the previous section, the first Chern class of the contact structure induced by $\alpha$ is zero and all Reeb orbits are closed. The description of the algorithm will use the notation introduced in Section 2. The proof is in Section 3.2. The algorithm works as follows.

(1) Compute the homology of $M$ using the algorithm of Randell [12] which we described in Section 2.2. This information can be used to determine more precisely what manifold $M$ is (if the dimension of $M$ is five, this step provides enough information to use the classification of Barden, see 11). This step also involves some numerics that are used in Step (4).

(2) Identify all orbit types that can occur for the Reeb flow. This is done as follows. For all subsets $I_{s} \subset I=\{0, \ldots, n\}, s>1$, the minimal positive time $T$ such that $\frac{2 T}{\pi}$ is divisible by all elements of $a_{i}, i \in I_{s}$, is $\frac{\pi}{2} \operatorname{lcm}_{i \in I_{s}}\left(a_{i}\right)$. The same minimal time $T$ can occur for several sets $I_{s}$. Let $J_{T}$ denote the largest such set. We obtain a collection of sets $J_{T_{1}}, \ldots, J_{T_{k}}$ for different $T_{1}, \ldots, T_{k}$. The corresponding submanifolds $M_{T_{i}}:=K\left(J_{T_{i}}\right)$ indicate submanifolds that are invariant under time $T_{i}$ of the Reeb flow. 
(3) Compute the Maslov indices of an orbit in $M_{T_{i}}$ with time $N T_{i}(N \in \mathbb{N})$. In order to ensure that we do not consider orbits of another orbit space, we have to choose $N$ such that for $i \neq j$ multiples $N T_{i}$ are not divisible by $T_{j}$ whenever $J_{T_{i}} \subset J_{T_{j}}$. Note that the Maslov index will only depend on the orbit type and not on the orbit itself. We may use the following formula

$$
\mu\left(S_{N T_{i}}\right)=2 \sum_{j \in J_{T_{i}}} \frac{2 N T_{i}}{\pi a_{j}}+2 \sum_{j \in I-J_{T_{i}}}\left\lfloor\frac{2 N T_{i}}{\pi a_{j}}\right\rfloor+\#\left(I-J_{T_{i}}\right)-4 N \frac{T_{i}}{\pi} .
$$

The algorithm fails if one does not obtain index positivity or negativity for the orbit spaces at this point. The conditions for these are given by

$$
\sum_{j=0}^{n} \frac{1}{a_{j}}>1 \text { for index positivity, and } \sum_{j=0}^{n} \frac{1}{a_{j}}<1 \text { for index negativity. }
$$

See also Remark 3.4 for a discussion if these conditions are not met.

(4) The dimension of the orbit space $S_{T_{i}}=M_{T_{i}} / S^{1}$ is given by $2 \# J_{T_{i}}-4$. Compute the rational homology of the orbit spaces $S_{T_{i}}$ in the following way. First of all, compute the rank of $\tilde{H}_{\# J_{T_{i}-2}}\left(M_{T_{i}}\right)$, given by

$$
\kappa=\operatorname{rk} \tilde{H}_{\# J_{T_{i}}-2}\left(M_{T_{i}}\right)=\sum_{I_{s} \subset J_{T_{i}}}(-1)^{\# J_{T_{i}}-s} \frac{\prod_{j^{\prime} \in I_{s}} a_{j^{\prime}}}{\operatorname{lcm}_{j \in I_{s}} a_{j}} .
$$

By our remark in Section 2.2 and Formula 2.5 we obtain

$$
H_{q}\left(S_{T_{i}}, \mathbb{Q}\right) \cong\left\{\begin{array}{l}
\mathbb{Q}, q \text { even, } 0 \leq q \leq \operatorname{dim} S_{T_{i}} \\
0, \text { otherwise }
\end{array}\right\} \oplus\left\{\begin{array}{l}
\mathbb{Q}^{\kappa}, q=\frac{1}{2} \operatorname{dim} S_{T_{i}} \\
0 \text { otherwise }
\end{array}\right\} .
$$

(5) The cylindrical contact homology with $\mathbb{Q}$-coefficients of $M$ with induced contact structure is a $\mathbb{Q}$-vector space, where the number of generators in each degree can be determined as follows. For each $T_{i}$ we get $\operatorname{rk} H_{j}\left(S_{T_{i}}, \mathbb{Q}\right)$ generators in degree $\mu\left(S_{N T_{i}}\right)+n-3+j-\frac{1}{2} \operatorname{dim} S_{T_{i}}$ for $j=0, \ldots, \operatorname{dim} S_{T_{i}}$ and $N \in \mathbb{N}$ such that for $j \neq i$ the multiples $N T_{i}$ are not divisible by $T_{j}$ whenever $J_{T_{i}} \subset J_{T_{j}}$. Here we use the Maslov-index that has been computed in Step 3.

For the cylindrical contact homology to be well defined and an invariant of the contact structure there should be no generators in degree $-1,0$ or 1 . To check this, one needs to define Morse functions $f_{T}$ on the orbit spaces $S_{T}$ following Bourgeois's construction described in Section 2.3. The critical points of these Morse functions form the Morse-Bott chain complex with grading given by Formula 2.7. If there are no critical points with degree $-1,0$ or 1 then the algorithm yields the cylindrical contact homology for contractible Reeb orbits. Note that these computations can depend on the choice of Morse functions.

Remark 3.1. We should emphasize at this point that cylindrical contact homology of Brieskorn manifolds is a periodic repetition of certain $\mathbb{Q}$ vector spaces with a degree shift. This can be seen as follows. Let us consider the orbit space $S_{T_{i}}$ and multiple coverings $S_{N T_{i}}$ where $N$ is chosen such that for $i \neq j$ the time $N T_{i}$ is not divisible by $T_{j}$ whenever $J_{T_{i}} \subset J_{T_{j}}$. If we add

$$
s_{i}:=\frac{\pi \operatorname{lem}_{j^{\prime} \in I} a_{j^{\prime}}}{2 T_{i}}
$$


to $N$, we remain in an orbit space of the same type as $\frac{\pi \mathrm{lcm}_{j^{\prime} \in I} a_{j^{\prime}}}{2}$ is divisible by all $T_{j}$. We see that the Maslov index changes as follows,

$$
\mu\left(S_{\left(N+s_{i}\right) T_{i}}\right)=\mu\left(S_{N T_{i}}\right)+2 \operatorname{lcm}_{j \in I} a_{j}\left(\sum_{j^{\prime}=0}^{n} \frac{1}{a_{j^{\prime}}}-1\right) .
$$

This shift of the Maslov index is independent of the orbit space $S_{T_{i}}$ and hence the terms in the contact homology corresponding to the orbit space $S_{T_{i}}$ are repeated with period at most

$$
2 \operatorname{lcm}_{j \in I} a_{j}\left(\sum_{j=0}^{n} \frac{1}{a_{j}}-1\right) .
$$

The periodicity of contact homology allows us to stop the algorithm after a finite number of steps.

Remark 3.2. Note that the requirement that $n \geq 3$, is not strictly necessary. For $n=2$ though, we are looking at 3 -dimensional Brieskorn manifolds, which are in general not simply connected. We can of course deal with these cases in an easy way by considering only contractible or homologically trivial Reeb orbits, but I have not worked out the details.

Remark 3.3. If we consider Brieskorn manifolds with large exponents, we can ensure that we have index negativity. In addition, large exponents ensure that the grading is strictly less than -1 , guaranteeing that cylindrical contact homology is well defined. Indeed if

$$
\min _{i \in I} a_{i} \geq \frac{5 n}{2}
$$

then the algorithm will always give the cylindrical contact homology of the Brieskorn manifold $\Sigma\left(a_{0}, \ldots, a_{n}\right)$. This estimate is rather rough and can be obtained from the formula for the Maslov index from Step (3).

Remark 3.4. One might wonder what happens if index positivity/negativity fails. This is the case if

$$
\sum_{j=0}^{n} \frac{1}{a_{j}}=1
$$

Note that in these cases there will always be degree 0 orbits. Indeed, putting the full period $T=\pi / 2 \mathrm{lcm}_{i \in I} a_{i}$ into Formula 3.1 yields Maslov index 0. By using the expression for the degree in part (5) of the algorithm we see that there will always be degree 0 orbits. This means that, for our choice of contact form, cylindrical contact homology is not well defined as an invariant.

\subsection{Examples.}

3.1.1. Cylindrical homology of some contact structures on $S^{2} \times S^{3}$. In this section we consider the family of Brieskorn manifolds of the form $M=\Sigma(2 l, 2,2,2)$ for $l>1$. Using our algorithm, it turns out that these manifolds are diffeomorphic to $S^{2} \times S^{3}$ and that their cylindrical contact homologies are all isomorphic. This is a bit exceptional, since typically we get very different homologies for different exponents. In the Section 4.1 we find some new exotic contact structures on spheres which illustrates the latter point. Let us now turn our attention to the application of the algorithm to $M=\Sigma(2 l, 2,2,2)$. The numbering is as in the algorithm. 
(1) We find $\operatorname{rk} H_{2}(M)=1$. Computation of the homology torsion by Randell's algorithm shows that there is none. By the classification of simply connected five manifolds [1] we see that $M \cong S^{2} \times S^{3}$, as the second StiefelWhitney class of $M$ is zero.

(2) The time $T_{1}=\frac{\pi}{2} 2$ is the minimal time for the sets which do not include 0 . Hence we see $J_{T_{1}}=\{1,2,3\}$.

The time $T_{2}=\frac{\pi}{2} 2 l$ appears as minimal time for the set $\mathrm{I}=\{0,1,2,3\}$. So $J_{T_{2}}=\{0,1,2,3\}$.

(3) We get for $N T_{1} \frac{2}{\pi}$ not divisible by $l$

$$
\mu\left(S_{N T_{1}}\right)=2 N+1+2\left\lfloor\frac{N}{l}\right\rfloor .
$$

The principal orbits have Maslov index

$$
\mu\left(S_{N T_{2}}\right)=2 l N+2 N .
$$

(4) We find $\operatorname{dim} S_{T_{1}}=2$ with $H_{0}\left(S_{T_{1}}, \mathbb{Q}\right)=\mathbb{Q}$ and $H_{2}\left(S_{T_{1}}, \mathbb{Q}\right)=\mathbb{Q}$. All other homology groups are 0 . The orbit space $S_{T_{2}}$ has dimension 4 , with the homology $H_{0}\left(S_{T_{2}}, \mathbb{Q}\right)=\mathbb{Q}, H_{2}\left(S_{T_{2}}, \mathbb{Q}\right)=\mathbb{Q}^{2}$ and $H_{4}\left(S_{T_{3}}, \mathbb{Q}\right)=\mathbb{Q}$. The other homology groups are zero.

(5) The period we defined in Remark 3.1 is equal to $2+2 l$. This allows us to compute fewer terms. For the first period, the contributions from $S_{N T_{1}}$ lie in degree

$$
2 N+2\left\lfloor\frac{N}{l}\right\rfloor+k
$$

for $N=1, \ldots, l-1$ (since we are considering a single period) and $k=0,2$. The contribution due to $S_{N T_{2}}$ are in degree

$$
2 l+k,
$$

for $k=0,2,4$. For the first period, we get one generator in degree 2, two generators in degree $4,6, \ldots, 2 l+2$ and one generator in degree $2 l+4$. The cylindrical contact homology has hence one generator in degree 2 and two generators in degree $2 k$ for $k>1$. We note that the cylindrical homology is well defined as there are no generators in degree $-1,0$ or 1 (lowest degree is higher than 1).

We can also apply the algorithm to $\Sigma(2,2,2,2)$, where there is just a single orbit space. This yields the same contact homology, see the discussion in 4.11 .

3.1.2. Some contact structures with index negativity. Let us consider Brieskorn manifolds with large exponents such that we have index negativity and that the degree is strictly less than -1 . In case all exponents are equal, contact homology is particularly easy to compute. For simplicity, we consider examples coming from Brieskorn manifolds of the form $M=\Sigma(k, k, k, k)$ with $k \geq 7$. Since $M$ has only a single orbit space, the computations are simple.

Step (1) shows that $\Sigma(k, k, k, k)$ is diffeomorphic to a connected sum of $k^{3}-$ $4 k^{2}+6 k-3$ copies of $S^{2} \times S^{3}$. The other steps yield the following. The minimal return time is $T=\frac{\pi}{2} k$. Hence we get the Maslov index

$$
\mu\left(S_{N T}\right)=2 \cdot 4 \cdot N-2 \cdot N \cdot k=2 N(4-k) .
$$


Step (4) of the algorithm shows that $H_{0}\left(S_{T} ; \mathbb{Q}\right) \cong \mathbb{Q}, H_{2}\left(S_{T} ; \mathbb{Q}\right) \cong \mathbb{Q}^{d}$, where $d=\kappa+1=\frac{(k-1)^{4}-1}{k}+2$. The last homology group is $H_{4}\left(S_{T} ; \mathbb{Q}\right) \cong \mathbb{Q}$. This gives a single period of contact homology. Taking the Maslov index into account we get the following for the contact homology of $M$.

We have one generator in degree $2 N(4-k)-2$ for $N=1,2, \ldots$ In degree $2 N(4-k)$ we have $\frac{(k-1)^{4}-1}{k}+2$ generators and in degree $2 N(4-k)+2$ we have again one generator $(N=1,2, \ldots)$.

3.2. Proof of the algorithm. Consider the Brieskorn manifold given by $M=$ $\Sigma\left(a_{0}, \ldots, a_{n}\right) \subset \mathbb{C}^{n+1}$ with contact form induced by $\alpha=\frac{i}{8} \sum a_{j}\left(z_{j} \mathrm{~d} \bar{z}_{j}-\bar{z}_{j} \mathrm{~d} z_{j}\right)$. The differential $\mathrm{d} \alpha=\frac{i}{4} \sum a_{j} \mathrm{~d} z_{j} \wedge \mathrm{d} \bar{z}_{j}$ is a symplectic form on $\mathbb{C}^{n+1}$. Let us denote this symplectic form by $\omega=\mathrm{d} \alpha$. Let $\xi$ be the contact structure given by $\left.\operatorname{ker} \alpha\right|_{M}$. Note that the contact form is of Morse-Bott type (Definition 2.1). This is seen as follows. Discreteness of the action spectrum is guaranteed by Step (2) of the algorithm. The sets $M_{T}$ in Definition 2.1 are Brieskorn manifolds with their standard contact form. In particular they are closed submanifolds of $M$. Note that this verifies the rank condition on $\mathrm{d} \alpha$ as well. The last condition for $\alpha$ being of Morse-Bott type can be checked by observing that the differential of the Reeb flow $\varphi$ is diagonal, see Formula 2.1 which allows us to verify the rank condition on $T \varphi-i d$.

We verify that Step (3) gives the correct Maslov indices. For each time $T_{i}$ that we found in Step (2) consider the $N$-fold covering of $M_{T_{i}}$ with $N$ such that $N T_{i}$ is not divisible by $T_{j}$ 's of a larger orbit space. Now let $p \in M_{T_{i}}$ and consider its orbit under the Reeb flow $\gamma(t):=\varphi_{t}(p)$ for $t \in\left[0, N T_{i}\right]$. To compute the indices we use Formula 2.3. We denote the obvious extension of the Reeb flow to $\mathbb{C}^{n+1}$ also by $\varphi_{t}$.

The symplectic action of the extended Reeb flow $\varphi_{t}$ on $\mathbb{C}^{n+1}$ is given by the differential of $T \varphi_{t}$, the diagonal matrix $\operatorname{diag}\left(e^{4 i t / a_{0}}, \ldots, e^{4 i t / a_{n}}\right)\left(t \in\left[0, N T_{i}\right]\right)$. As in Section 2.1 we denote the path of symplectic matrices induced by the extended flow by $\Phi_{\mathbb{C}^{n+1}}$. We can now use the additivity of the Maslov index and Formula 2.4 to get the index of this path. This gives us

$$
\mu\left(\Phi_{\mathbb{C}^{n+1}}\right)=2 \sum_{a_{j} \in J_{T_{i}}} \frac{2 N T_{i}}{\pi a_{j}}+2 \sum_{a_{j} \in I-J_{T_{i}}}\left\lfloor\frac{2 N T_{i}}{\pi a_{j}}\right\rfloor+\#\left(I-J_{T_{i}}\right) .
$$

With respect to the symplectic basis of $\xi^{\omega}$ found in Section 2 the action induced on $\xi^{\omega}$ by the extended Reeb flow is given by Formula 2.2. As before we use the notation from Section 2.1, i.e. $\Phi_{\xi \omega}$ denotes the path of symplectic matrices induced by the action of the extended Reeb flow. Formula 2.4 gives us

$$
\mu\left(\Phi_{\xi \omega}\right)=4 N \frac{T_{i}}{\pi} .
$$

Taking the difference of these Maslov indices yields the desired result from Step (3). The conditions for index positivity and negativity can be found by observing that $\lfloor t\rfloor \geq t-1$ and $\lfloor t\rfloor \leq t$.

We show that the determinant bundle of the linearized Cauchy-Riemann operator can be oriented directly, i.e. we shall show that the linearized Cauchy-Riemann operator is asymptotic to a complex linear operator. First of all, we note that near the punctures this operator can be given the form (see for instance 2], chapter 3 and 5)

$$
\frac{\partial}{\partial s}+J_{0} \frac{\partial}{\partial t}+S(s, t)
$$


where $S$ are symmetric matrices and $J_{0}$ is the standard complex structure and $(s, t)$ are cylindrical coordinates near the puncture, $t$ for the $S^{1}$ direction. We have

$$
\lim _{s \rightarrow \infty} S(s, t)=\tilde{S}(t)
$$

with

$$
\tilde{S}(t)=-J_{0} \frac{d \psi(t)}{d t} \psi^{-1}(t)
$$

where $\psi$ are the symplectic matrices obtained from the linearized Reeb flow in the symplectization $M \times \mathbb{R}$. Note that if these matrices $\psi(t)$ are unitary with respect to the above trivialization, the matrix $\tilde{S}$ will commute with $J_{0}$. We will verify that we can choose a trivialization such that the matrices $\psi$ are unitary.

Note that the linearized extended Reeb flow on $\mathbb{C}^{n+1}$ is represented by a path of unitary matrices, see Section 2.1. Keep in mind that the metric is given by $\omega(\ldots, J \ldots)$, where $J$ is the standard complex structure on $\mathbb{C}^{n+1}$. For the above purposes we need a trivialization that comes from the symplectization of $M$, so this one is not suitable. To that end, recall that a contact structure $\xi$ is symplectically stably trivial if and only if $\xi \oplus \mathbb{C}$ is symplectically trivial (see also Remark 4.2). This means that we can split off a complex line bundle from our trivialization on $\mathbb{C}^{n+1}$. Define $V:=\operatorname{span}_{\mathbb{R}}\left(\tilde{X}_{1}, \tilde{Y}_{1}\right)$ using the notation from Section 2 and let $W$ be the orthogonal complement of $V$ in $\mathbb{C}^{n+1}$. Because the linearized extended Reeb flow maps $V$ to $V$ (see Section 2.1), we can see that the linearized extended Reeb flow induces a map from $W$ to $W$ which is unitary with respect to the induced metric. Note also that $\xi$ is a subbundle of $W$ and $W$ contains the Reeb line bundle. Hence $W$ can be identified with the tangent bundle of the symplectization of $M$ restricted to $M \times\{$ point . Let $\psi(t)$ be the path of unitary matrices given by the linearized extended Reeb flow on $W$ with respect to an orthonormal basis of $W$.

If we define $\tilde{S}(t)$ as above in terms of the unitary $\psi(t)$, then it follows that the Cauchy-Riemann operator

$$
\frac{\partial}{\partial s}+J \frac{\partial}{\partial t}+\tilde{S}(t)
$$

is complex linear. By the above discussion, this operator is asymptotic to the given linearized Cauchy-Riemann operator on the symplectization. As mentioned in Section 2.3, this gives us an orientation of the determinant bundle. In particular, no bad orbits or disorienting loops can occur.

Index positivity/negativity is verified in Step (3) of the algorithm. If we have that there are no generators of degree $-1,0$ or 1 , then the homology of the MorseBott complex is isomorphic to cylindrical contact homology according to Theorem 2.6. Consider the Morse-Bott complex with generators the critical points $p$ of the chosen Morse functions with grading given by $\mu\left(S_{T}\right)-\frac{1}{2} \operatorname{dim} S_{T}+\operatorname{index} p+n-3$. The differential of the Morse-Bott complex is given by Formula 2.8. The differential acting on $p \in S_{T}$ is given by

$$
d p=\partial p+\sum n_{q} q
$$

The first term is the Morse-Witten differential for the critical points of the Morse functions $f_{T}$. The second term counts the number of elements in the zero-dimensional part of the fibered product

$$
\left(W^{u}(p) \times_{S} \mathcal{M}^{f_{T}}\left(S ; S^{\prime}\right) \times_{S^{\prime}} W^{s}(q)\right) / \mathbb{R} .
$$


Now note that there is an $S^{1}$-action on the symplectization of $M$ induced by the Reeb flow. A holomorphic curve asymptotic to closed Reeb orbits comes therefore in at least an $S^{1}$-family (by letting the Reeb flow act) except in the case that the curve is a vertical cylinder. This means the above fibered product is at least 1 dimensional, so it will not contribute to the differential. This argument is also used in Section 9.3 of [2].

This implies that the only non-zero contribution to the differential comes from $\partial p$, which means that the cylindrical contact homology is isomorphic to the MorseWitten homology of the orbit spaces $S_{T}$ with degree shifts of $\mu\left(S_{T}\right)-\frac{1}{2} \operatorname{dim} S_{T}+$ $n-3$. As the Morse-Witten homology of the orbit spaces is equal to the rational homology of the orbit spaces which is computed in Step (4), we find that the contact homology is given by our algorithm.

\section{Exotic contact Structures}

Our aim in this section is to describe a certain class of contact manifolds that admits infinitely many non-isomorphic contact structures.

Given two contact manifolds $\left(M_{1}, \xi_{1}\right)$ and $\left(M_{2}, \xi_{2}\right)$, we can build a new contact manifold by forming their connected sum, 11] and [16. If we think of a connected sum as first removing a disk from both $M_{1}$ and $M_{2}$ and then gluing them via a "connecting" tube, then the contact structure on $M_{1} \# M_{2}$ can be be made to coincide with the contact structure on $M_{1}$ with a disk removed and $M_{2}$ with a disk removed.

In order to say something about the contact homology of the connected sum, we find generic contact forms $\alpha_{1}, \alpha_{2}$ for the contact structures $\xi_{1}$ and $\xi_{2}$, i.e. contact forms whose closed Reeb orbits are non-degenerate.

First we need another, but similar notion of index positivity, which we will refer to as Ustilovsky index positivity. Suppose that a contact structure $\xi$ is symplectically stably trivial and let $F$ be a corresponding trivialization. We may then compute the Maslov index with respect to the trivialization $F$. The index does depend on the trivialization and we indicate this by making the Maslov index visibly dependent on the trivialization $F$ by writing $\mu(\ldots ; F)$. We use $\varepsilon$ to indicate the trivial bundle.

Definition 4.1. Let $(M, \alpha)$ be a contact manifold. Assume that $\pi_{1}(M)=0$ and that the bundle $(\xi, d \alpha)$ is symplectically stably trivial. Let $F$ be a symplectic trivialization of $\xi \oplus \varepsilon^{2}$. The manifold $(M, \alpha)$ is called Ustilovsky index-positive if there exist constants $c>0$ and $d$ such that for any Reeb orbit $\gamma$

$$
\mu(\gamma ; F) \geq c \mathcal{A}(\gamma)+d
$$

holds.

Remark 4.2. Ustilovsky has shown in his thesis that a contact structure $\xi$ is symplectically stably trivial if and only if $\xi \oplus \varepsilon^{2}$ is trivial and that the notion of Ustilovsky index positivity does not depend on the choice of trivialization. Note that the Chern class of a symplectically stably trivial contact structure is trivial.

Example 4.3. Brieskorn manifolds are symplectically stably trivial (for instance, see Section (2). Hence we may consider Ustilovsky index positivity of a Brieskorn manifold $\Sigma\left(a_{0}, \ldots, a_{n}\right)$. Note that a Brieskorn manifold has Ustilovsky index positivity if all orbits types have index positivity. Namely, our computation of the 
Maslov indices for closed orbits used the trivialization coming from $\mathbb{C}^{n+1}$. If use that trivialization to verify Ustilovsky index positivity, we see that the formula from Step (3) of the algorithm can be modified as follows

$$
\mu\left(\left.\gamma(t)\right|_{t \in[0, T]}\right)=\sum_{j=0}^{n} \mu\left(\left.e^{4 i t / a_{j}}\right|_{t \in[0, T]}\right)-\mu\left(\left.e^{4 i t}\right|_{t \in[0, T]}\right)
$$

where the Reeb orbit $\gamma$ is given by

$$
\gamma(t)=\left(e^{4 i t / a_{0}} z_{0}, \ldots, e^{4 i t / a_{n}} z_{n}\right),
$$

for $\left(z_{0}, \ldots, z_{n}\right) \in \Sigma\left(a_{0}, \ldots, a_{n}\right) \subset \mathbb{C}^{n+1}$. Then apply Formula 2.4.

We have the following theorem from Ustilovsky ([15], Theorem 5.2.1):

Theorem 4.4 (Ustilovsky). Let $\left(M_{1}, \alpha_{1}\right),\left(M_{2}, \alpha_{2}\right)$ be two simply connected contact manifolds of dimension $2 n-1$ that have Ustilovsky index positivity. Assume all periodic orbits of $R_{\alpha_{1}}$ and $R_{\alpha_{2}}$ are non-degenerate. Then for any integer $N$ there exists a contact form $\alpha$ on $M=M_{1} \# M_{2}$ so that

(1) $(M, \alpha)$ is Ustilovsky index-positive.

(2) All periodic orbits of $R_{\alpha}$ are non-degenerate.

(3) If $c_{j}^{1}, c_{j}^{2}$ and $c_{j}$ denote the numbers of periodic Reeb orbits of degree $j$ in $M_{1}, M_{2}$ and $M$, respectively, then for $j \leq N$, we have $c_{j}=c_{j}^{1}+c_{j}^{2}+\beta_{j}$, where $\beta_{j}=1$ for $j=2 n-3,2 n-1, \ldots$ and $\beta_{j}=0$ otherwise.

The $\beta_{j}$ 's in this theorem are the degrees of the periodic Reeb orbits in the connecting tube. We will take for $M_{1}$ any contact manifold satisfying the conditions of the above theorem of Ustilovsky and for $M_{2}$ we will take a special contact sphere. This sphere will have the property that its contact homology contains generators with degree lower than the lowest degree of a generator in the connecting tube. After taking the connected sum with $M_{1}$ and using Ustilovsky's theorem, the resulting contact manifold $M_{1} \# M_{2}$ will be diffeomorphic to $M_{1}$, but its cylindrical contact homology will have more generators in low degrees.

4.1. Construction of a special contact sphere. Let us consider the Brieskorn manifold $M=\Sigma\left(p_{1}, \ldots, p_{n-1}, 2,2\right)$ where $p_{1}, \ldots, p_{n-1}$ are odd primes which will be specified later (they need to be chosen large enough). Notice that we immediately see that this manifold is homeomorphic to a sphere by Theorem 14.5 in 8 . This fact can also be seen by computing the homology of $M$ using Randell's algorithm. Then use the fact that Brieskorn manifolds of dimension at least 5 are always simply connected and conclude that $M$ is homeomorphic to a sphere with the generalized Poincaré conjecture as proved by Smale. We apply our algorithm to compute the first terms of the contact homology.

4.1.1. Contact homology of $\Sigma\left(p_{1}, \ldots, p_{n-1}, 2,2\right)$. We have the following orbit types and Maslov indices.

- $I_{2}=\{2,2\}$. The minimal return time for orbits of this type is $T=\pi$. The Maslov index of the corresponding orbits is given by

$$
\mu\left(S_{N T}\right)=2 \sum_{i=1}^{n-1}\left\lfloor\frac{2 N}{p_{i}}\right\rfloor+n-1 \geq n-1
$$

If the $p_{i}$ 's are odd primes, the first term will vanish for at least $N=1$. Now we turn our attention to the homology of the orbit space. It has dimension 
0 and Formula 2.5 shows that $H_{0}\left(S_{T}, \mathbb{Q}\right)=\mathbb{Q}^{2}$. This shows that the contact homology of $M$ has at least 2 generators in degree $2 n-4$. Since the first term is always even, multiple covers of this orbit will have either the same degree or a higher even degree. Note that this orbit type will not give any generators in degree $2 n-3$.

- Sets of the form $\left\{p_{i_{1}}, \ldots, p_{i_{k}}\right\}$ with $k$ at least 2 . The minimal return time is now $T=p_{i_{1}} \cdot \ldots \cdot p_{i_{k}} \frac{\pi}{2}$. The Maslov indices of the orbit spaces are given by

$$
\begin{aligned}
\mu\left(S_{N T}\right) & =2 \sum_{j} p_{i_{1}} \cdot \ldots \cdot \hat{p}_{i_{j}} \cdot \ldots \cdot p_{i_{k}} N+4\left\lfloor\frac{N p_{i_{1}} \cdot \ldots \cdot p_{i_{k}}}{2}\right\rfloor \\
& +2 \sum_{l \neq i_{j}}\left\lfloor\frac{N p_{i_{1}} \cdot \ldots \cdot p_{i_{k}}}{p_{l}}\right\rfloor+n-1-k+2-2 N p_{i_{1}} \cdot \ldots \cdot p_{i_{k}} .
\end{aligned}
$$

Note that $4\left\lfloor\frac{N p_{i_{1}} \cdot \ldots \cdot p_{i_{k}}}{2}\right\rfloor \geq 2 N p_{i_{1}} \cdot \ldots \cdot p_{i_{k}}-2$ and that $\operatorname{dim} S_{T}=2 k-4$. Using this estimate, we find that the degree of the associated generators can be estimated from below as

$$
\text { degree } \geq 2 n-2-2 k+2 \sum_{j} p_{i_{1}} \cdot \ldots \cdot \hat{p}_{i_{j}} \cdot \ldots \cdot p_{i_{k}} N .
$$

Since the sum contains at least two terms, we can make the Maslov index arbitrarily large by choosing big primes. In particular, the degree of these orbit types can be assumed to be larger than $2 n-3$.

- Sets of the form $\left\{p_{i_{1}}, \ldots, p_{i_{k}}, 2,2\right\}$. The minimal return time is $T=p_{i_{1}}$. $\ldots \cdot p_{i_{k}} \pi$. The associated Maslov indices are

$$
\mu\left(S_{N T}\right)=4 \sum_{j} p_{i_{1}} \ldots \hat{p}_{i_{j}} \cdot \ldots \cdot p_{i_{k}} N+2 \sum_{l \neq i_{j}}\left\lfloor\frac{2 N p_{i_{1}} \cdot \ldots \cdot p_{i_{k}}}{p_{l}}\right\rfloor+n-1-k .
$$

For $k>1$ the first term will contain at least one $p_{i}$-term. This means that the degree (note that the dimension of the orbit space is now $2 k$ ) will become as large as we like by choosing the $p$ 's accordingly. For $k=1$ the first term is $4 N$ and we see that the degree is at least $4 N+n-3+n-3 \geq 2 n-2$.

Summarizing these estimates, we see that by choosing suitable primes, we may assume that the contact homology contains at least two generators in degree $2 n-4$ and no generators in degree $2 n-3$. Note that we have (Ustilovsky) index positivity since two exponents are 2, see the condition from Step (3) of the algorithm.

In order to be able to apply Theorem 4.4 we need to have a generic contact form on $M$ that has at least two closed Reeb orbits in degree $2 n-4$ and no generators in degree $2 n-3$. We will do this, along with a more general statement in the following interlude.

4.1.2. Generic contact forms. In this section, we want to associate a generic contact form, i.e. a contact form whose closed Reeb orbits are non-degenerate, to the MorseBott contact forms used in our algorithm.

Let $M$ be a contact manifold of dimension $2 n-1$ and let $\alpha$ be a contact form on $M$ that satisfies the Morse-Bott condition. We use Bourgeois's construction of Morse functions on the orbit spaces described in Section 2.3. We use his ideas to perturb $\alpha$ into a generic contact form such that we still have some information on 
the indices of the closed Reeb orbits. The following observation by Ustilovsky [15] will play a key role.

Lemma 4.5. If $(M, \alpha)$ is Ustilovsky index-positive, then a $C^{\infty}$-small perturbation $\alpha^{\prime}$ of $\alpha$ the manifold $\left(M, \alpha^{\prime}\right)$ is also Ustilovsky index-positive. Moreover, if $\mu(\gamma ; F) \geq c \mathcal{A}(\gamma)+d$ for orbits $\gamma$ of $R_{\alpha}$ then, for $\alpha^{\prime}$ close enough to $\alpha, \mu\left(\gamma^{\prime} ; F^{\prime}\right) \geq$ $c^{\prime} \mathcal{A}\left(\gamma^{\prime}\right)+d^{\prime}$ for orbits $\gamma^{\prime}$ of $R_{\alpha^{\prime}}$, where $c^{\prime}=c / 2$ and $d^{\prime}=-|d|-2 n$.

Remark 4.6. Note that for a small perturbation, we have a one-to-one correspondence between non-degenerate Reeb orbits of the contact form and of the perturbed contact form up to some period. The Conley-Zehnder indices of the corresponding orbits are the same.

Lemma 4.7. Let $\alpha$ be the standard contact form on the Brieskorn manifold $M=$ $\Sigma\left(a_{0}, \ldots, a_{n}\right), n \geq 3$. Assume that the exponents are such that we have Ustilovsky index positivity (cf. Example 4.3). Then for all $N \in \mathbb{Z}$ there exists a generic contact form $\alpha^{\prime}$, such that all generators of the chain complex of $\alpha^{\prime}$ coincide with the generators of the Morse-Bott chain complex of $\alpha$ up to degree $N$.

Proof. Let us denote the constants from the Ustilovsky index positivity by $c>0$ and $d$ such that $\mu(\gamma) \geq c \mathcal{A}(\gamma)+d$ for a part $\gamma$ of a Reeb orbit. Choose $T \geq$ $\max \left\{N, \frac{4}{c}(N+|d|+4 n)\right\}$. By Lemma 2.2 we find a perturbation $\alpha^{\prime \prime}$ of $\alpha$ such that its periodic Reeb orbits up to action $T$ are non-degenerate and correspond to critical points of the chosen Morse functions on the orbit spaces. Since we have Ustilovsky index positivity for $\alpha$, we will have the same for $\alpha^{\prime \prime}$ by Lemma 4.5 where the constants are now $c / 2$ and $-|d|-2 n$.

We perturb $\alpha^{\prime \prime}$ further to make all Reeb orbits non-degenerate and call the perturbation $\alpha^{\prime}$. Once again $\alpha^{\prime}$ is Ustilovsky index positive with constants $c / 4$ and $-|d|-4 n$. By Remark 4.6 this perturbation will not change the Conley-Zehnder indices of orbits with period up to $T$ (and in particular up to $N$ ). The perturbation will introduce new periodic Reeb orbits. However, the newly created ones can be made to have period larger than $T$ (by making a small enough perturbation). As a result of Lemma 4.5, their Conley-Zehnder indices will be larger than $T c / 4-|d|-$ $4 n \geq N$. This proves our lemma.

4.1.3. A generic contact form for $\Sigma\left(p_{1}, \ldots, p_{n-1}, 2,2\right)$. We continue with our construction of a contact sphere from Section 4.1. As before, we write our Brieskorn sphere as $M=\Sigma\left(p_{1}, \ldots, p_{n-1}, 2,2\right)$. We get the Morse-Bott chain complex for contact homology by following Bourgeois's construction of Morse functions for the orbit spaces from Section 2.3. Note that the computation of the contact homology of $M$ in Section 4.1.1 shows that the Morse-Bott complex will have at least two generators in degree $2 n-4$ and no generators in degree $2 n-3$. This holds true because the lowest dimensional orbit spaces have dimension 0 , so the number and degree of generators associated to those orbit spaces do not depend on the choice of Morse functions. Note that these data do depend on the choice of Morse functions for the other orbit spaces.

Now we apply Lemma 4.7 to obtain a generic contact form where the the number and degree of generators of the chain complex coincide with those of the Morse-Bott complex up to degree $2 n-2$. This gives $M$ a generic contact form and allows us to use Theorem 4.4 


\subsection{Constructing new contact structures.}

Theorem 4.8. Let $(M, \xi)$ be a simply-connected contact manifold. Assume furthermore that $M$ admits a nice contact form (a contact form without any closed Reeb orbits of degree $-1,0$ or 1) that has Ustilovsky index positivity (in particular $\left.c_{1}(\xi)=0\right)$. Then $M$ admits infinitely many non-isomorphic contact structures.

Proof. Let $N^{\prime}=\Sigma\left(p_{1}, \ldots, p_{n-1}, 2,2\right)$. The above discussion shows that $N^{\prime}$ admits a generic contact form that is Ustilovsky index positive with at least two generators in degree $2 n-4$, no generators in degree $2 n-3$ and no generators in lower degrees. As remarked before, we know that $N^{\prime}$ is homeomorphic to a sphere (Theorem 14.5 in [8). Since the differentiable structures on a sphere form a finite group, we can find an $r$ such that $N:=\underbrace{N^{\prime} \# \ldots \# N^{\prime}}_{r}$ is diffeomorphic to the standard sphere. By Theorem 4.4, $N$ admits a generic contact form whose cylindrical contact homology has at least $2 r$ generators in degree $2 n-4$, precisely $r-1$ generators in degree $2 n-3$ and no generators in lower degrees.

Now apply Theorem 4.4 to $M$ and $N$. The connected sum $M \# N$ will be diffeomorphic to $M$, since $N$ is diffeomorphic to $S^{2 n-1}$. The theorem shows that the connected sum still admits a nice contact form (because no generators are added in degrees $-1,0$ or 1$)$. The number of generators of the chain complex of the cylindrical contact homology is increased by at least $2 r$ in degree $2 n-4$ and by $r$ in degree $2 n-3$. Because of Ustilovsky index positivity there are only a finite number of generators in each degree. Since the number of generators in degree $2 n-5$ is unchanged by taking the connected sum with $N$, taking repeated connected sums with $N$ will ensure that we get a contact structure on $M$ with a contact homology different from the original one, namely with more generators in degree $2 n-4$. By taking more connected sums with $N$ we get infinitely many contact structures on $M$, all with different cylindrical contact homology distinguished by the rank of the homology in degree $2 n-4$.

Remark 4.9. Note that if two contact manifolds satisfy the conditions of Theorem 4.8, their connected sum satisfies those conditions as well by Theorem 4.4 In addition, any index positive Brieskorn manifold whose cylindrical contact homology can be computed using our algorithm satisfies the conditions of Theorem 4.8. This can be seen by applying Lemma 4.7

As an example, the cylindrical contact homology of $\Sigma(2, \ldots, 2)=S T^{*} S^{n}$ can be computed using our algorithm (the computation is similar to the one in Section 3.1.2), which shows that $\#_{k} S T^{*} S^{n}$ carries infinitely many contact structures for all $k \in \mathbb{N}$ and $n>2$.

Question 4.10. Consider the orbifold $X_{a}=\Sigma\left(a_{0}, \ldots, a_{n}\right) / S^{1}$. We can compute the orbifold cohomology of $X_{a}$ following Chen and Ruan, see [3]. This is done by taking the direct sum of the homologies of the singular subspaces with appropriate degree shifts. This is similar to what we do in our algorithm, where our "degree shifts" are expressed in the Maslov indices.

Orbifold cohomology is not quite the same as contact homology, though. First of all, contact homology is periodic, whereas orbifold cohomology has only non-trivial groups in a finite number of degrees. Secondly, the orbifold cohomology can have rational grading and contact homology has integer grading in our cases. 
A single period of contact homology could however be related to orbifold cohomology. Indeed, in case $X_{a}$ is a manifold and not just an orbifold (for instance when all exponents of the Brieskorn manifold are equal), contact homology can be expressed as a periodic repetition of the homology of $X_{a}$. In general, the degree shifts of orbifold cohomology do not match the ones coming from the Maslov index. We can, of course, transform the degree shifts to correct this. Can this be done in a meaningful way? This might give some insight of how to compute contact homology for more general $S^{1}$-bundles over symplectic orbifolds.

Question 4.11. In 9] Lerman gives a description of a number of contact structures on $S T^{*} S^{3} \cong S^{2} \times S^{3}$. Some are distinguished by their first Chern class, but he is also able to produce many contact structures with the same Chern class. One can wonder whether these contact structures are isomorphic. The contact structures we found in 3.1.1 are related to this example. Indeed, Lerman produces infinitely many contact structures in each even first Chern class except when $c_{1}$ is zero. This is complemented by the Brieskorn manifolds $\Sigma(2 k, 2,2,2)$ which all have trivial Chern class. Note however that the cylindrical contact homology is not able to distinguish between those contact structures. This leaves the question whether one is able to distinguish these possibly non-isomorphic contact structures in each even Chern class.

Question 4.12. Can one show the result of Theorem 4.8 under milder conditions? In particular, to what extend is index positivity necessary?

\section{REFERENCES}

[1] Barden, D.: Simply connected five-manifolds. Ann. of Math. (2) 82 (1965), 365-385

[2] Bourgeois, F.: A Morse-Bott approach to contact homology. $\mathrm{PhD}$ thesis, Stanford University, 2002

[3] Chen, W., Ruan, Y.: A New Cohomology Theory for Orbifold. Comm. Math. Phys. 248 (2004), no. 1, 1-31

[4] Eliashberg, Y.: On symplectic manifolds with some contact properties. J. Differential Geom. 33 (1991), no. 1, 233-238

[5] Eliashberg, Y.: Invariants in contact topology. Proceedings of the International Congress of Mathematicians, Vol. II (Berlin, 1998). Doc. Math. 1998, Extra Vol. II, 327-338 (electronic)

[6] Eliashberg, Y., Givental, A., Hofer, H.: Introduction to symplectic field theory. GAFA 2000 (Tel Aviv, 1999). Geom. Funct. Anal. 2000, Special Volume, Part II, 560-673

[7] Giroux, E.: Une structure de contact, même tendue, est plus ou moins tordue. Ann. Sci. École Norm. Sup. (4) 27 (1994), no. 6, 697-705

[8] Hirzebruch, F., Mayer, K. H.: $\mathrm{O}(n)$-Mannigfaltigkeiten, exotische Sphären und Singularitäten. Lecture Notes in Mathematics, No. 57. Springer-Verlag, Berlin, 1968

[9] Lerman, E.: Maximal tori in the contactomorphism groups of circle bundles over Hirzebruch surfaces. Math. Res. Lett. 10 (2003), no. 1, 133-144

[10] Lutz, R., Meckert, C.: Structures de contact sur certaines sphères exotiques. C. R. Acad. Sci. Paris Sér. A-B 282 (1976), no. 11, Aii, A591-A593

[11] Meckert, C.: Forme de contact sur la somme connexe de deux variétés de contact de dimension impaire.

Ann. Inst. Fourier (Grenoble) 32 (1982), no. 3, xi, 251-260

[12] Randell, R. C.: The homology of generalized Brieskorn manifolds. Topology 14 (1975), no. 4, 347-355 
[13] Robbin, J., Salamon, D.: The Maslov index for paths. Topology 32 (1993), no. 4, 827-844

[14] Ustilovsky, I.: Infinitely many contact structures on $S^{4 m+1}$. Internat. Math. Res. Notices (1999), no. 14, 781-791

[15] Ustilovsky, I.: Contact homology and contact structures on $S^{4 m+1}$. $\mathrm{PhD}$ thesis, Stanford University, 1999

[16] Weinstein, A.: Contact surgery and symplectic handlebodies. Hokkaido Math. J. 20 (1991), no. 2, 241-251

Otto van Koert, Université Libre de Bruxelles, Département de Mathématiques CP 218, Boulevard du Triomphe, B-1050 Bruxelles - Belgique

E-mail address: ovkoert@ulb.ac.be 